\title{
Mathematical Models for Microparasites of Wildlife
}

\section{J.A.P. Heesterbeek and M.G. Roberts}

\section{Introduction}

In this chapter we review some of the basic theory of deterministic modelling of microparasitic diseases of wild animal populations. As discussed by Dobson and Grenfell in the Introduction to this volume, the special feature of wildlife diseases is that host population size is a dynamic variable. This requires significant modifications to classical epidemic theory based on constant host populations. Particularly since the seminal work of Anderson and May (1979), there has been a large body of research in this area (reviewed by Barlow, Begon and Bowers, Briggs et al. (this volume)). Here we review some basic issues in the modelling of microparasitic infections in naturallyfluctuating animal populations. The paper is not intended as an in depth study of microparasite models, but should be seen more as a summer exhibition at a museum with little bits of everything, as a guide to the art of elementary modelling and the less accessible more mathematical literature where the exhibits are usually stored.

There are many purposes for using mathematical models in epidemiology (see Anderson and May (1991), Hethcote and Van Ark (1992) for an extensive list). Among the main 'justifications' are, firstly, the fact that models provide insight into the relative importance of factors that influence spread, and improve our understanding of the relationship between mechanisms that operate on the level of the individual and the phenomena that result on the population-level (after Diekmann 1991). Secondly, the formulation of mathematical models requires precision about the underlying hypothesis and could make one aware of working hypotheses that might otherwise go unnoticed and which, once discovered, might deserve scrutiny. The analysis of mathematical models can lead to the introduction of new concepts that turn out to play an vital role. The most important example of this is the idea of a threshold quantity for epidemic development (the basic reproduction ratio $\left.R_{0}\right)$. Another use of models is in clarifying which parameters are critical in their influence on the dynamical behaviour. This may lead to the discovery of key-parameters whose numerical (range of) value(s) could still be unknown. Finally, we mention the dominant use of models in performing thought experiments with respect to, for example, the evaluation of the efficacy of given 
(or even still imagined) control measures, in cases where actual experiments are impossible because of practical, ethical or economic constraints.

The main use of mathematical models is, perhaps surprisingly, not in predicting future trends. One of the reasons for this is that the most complex models for specific diseases are still (highly) oversimplified. Furthermore, our knowledge of the values of many parameters in the transmission process is often poor and making models more complex rapidly leads to a proliferation of these parameters, the values of which are difficult to determine with accuracy. Accurate quantitative predictions are therefore practically impossible, particularly given the potential complexities of dynamic wildlife host populations noted above.

In Section 2 of this paper, we introduce some simple concepts from epidemic theory. Starting with the general approaches of Kermack and McKendrick (1927) and Ross and Hudson (1917), we specialise to the compartmental approach to disease modelling that is most prominent in the literature today. We discuss concepts such as invasion (connected to the basic reproduction ratio $R_{0}$ ), epidemic, final size, endemic state, and control. The general Kermack and McKendrick model and the way that compartmental models are contained in it as special cases, are treated in Appendix A. In Section 3, we first introduce a simple (but not too simple) basic compartmental model, which we then proceed to extend in various directions, describing the dynamic behaviour of the models so obtained. The impact of host population dynamics is a particular focus here. The evaluation of control strategies such as vaccination and culling is discussed, with reference to the basic model, in Section 4. In Section 5, we briefly study the inclusion of heterogeneity in infection rates in the model, and finally, Appendix B is devoted to the definition and calculation of $R_{0}$ in heterogeneous populations.

Even the largest summer exhibition cannot cover all aspects of art, and happily there is no need for this because summer comes to museums everywhere. There are, first, a number of general useful books devoted to epidemiological modelling. In order of appearance we mention Bailey (1975) (stochastic aspects), Anderson (1982b) (a collection of papers, partly on human diseases, partly on animal diseases), Hethcote and Yorke (1984) (gonorrhea, but with much insight to modelling infectious diseases in general), Becker (1989) (data analysis), Anderson and May (1991) (a major tome, providing many insights on human disease epidemiology) and Busenberg and Cooke (1993) (a good introduction to mathematical modelling of infectious diseases, much broader than its title suggests). We do not discuss stochastic models, although we briefly go into stochasticity connected with fade-out in Section 2.4 (see also the Dye et al. this volume). Stochastic models of wildlife microparasites are mainly concerned with the spatial spread of rabies (see e.g. papers by Ball, Mollison and others in Bacon (1985)). For recent 
advances in stochastic modelling of infectious diseases we refer to Gabriel et al. (1990), Mollison et al. (1991) and Gabriel et al. (1993). On a smaller scale but mainly devoted to wildlife diseases, we refer to Mollison and Levin (this volume), Bolker et al. (this volume) and Swinton and Anderson (this volume) for aspects related to spatial spread of animal diseases. For an excellent review of the modelling of spatial spread see Metz and Van den Bosch (1995). For vector transmitted diseases we refer to Dye and Williams (this volume), and for multi-host/multi-parasite interactions see Begon and Bowers (this volume). Finally, see Barlow (this volume) for an extensive critical evaluation of models for wildlife microparasitic diseases.

\section{Some elementary epidemic theory}

An important characteristic of the class of disease-causing organisms referred to as 'microparasites' is that, once infection has taken place, the disease develops within the body of the host individual without influence from the external environment (Dobson and Hudson this volume). In general, the microparasites reproduce within the host's body at such a high rate that any additional infection by the same parasite will go unnoticed. This observation is due to Ross and Hudson (1917) and Kermack and McKendrick (1927) in their seminal contributions to the mathematical theory of epidemics. Following this observation, both papers essentially describe the expected infectivity of an infected individual as a function $\mathcal{A}(\tau)$ of $\tau$, the time elapsed since that individual became infected. The idea is that $\mathcal{A}$ is the average taken over all possible stochastic time evolutions of the internal population of microparasites and the concomitant reaction occuring in the immune system of the host. The precise nature of $\mathcal{A}$ is, of course, determined by the particulars of the disease under study. In Figure 1, we give two examples of this function $\mathcal{A}$. The first graph represents a herpes infection in (wild)fowl (with recrudescence in carriers at irregular intervals after $6-8$ weeks), the second graph represents rabies in foxes (both graphs are from G. Smith, personal communication). Note the difference in time-scale between the two examples.

In this paper we will focus on the more familiar compartmental models because they are the most frequently used in the literature. A concise description, connecting the more flexible infectivity function approach with compartmental models of disease progression, is given in Appendix A. For a recent review of mathematical modelling involving the infectivity function $\mathcal{A}(\tau)$ see Diekmann et al. (1995). For a recent review of compartmental models for infectious diseases we refer to Anderson and May (1991), Hethcote (1994).

In the compartmental approach, one generally distinguishes four classes, the susceptible individuals $S$, the latent individuals $E$ (i.e. infected individuals 

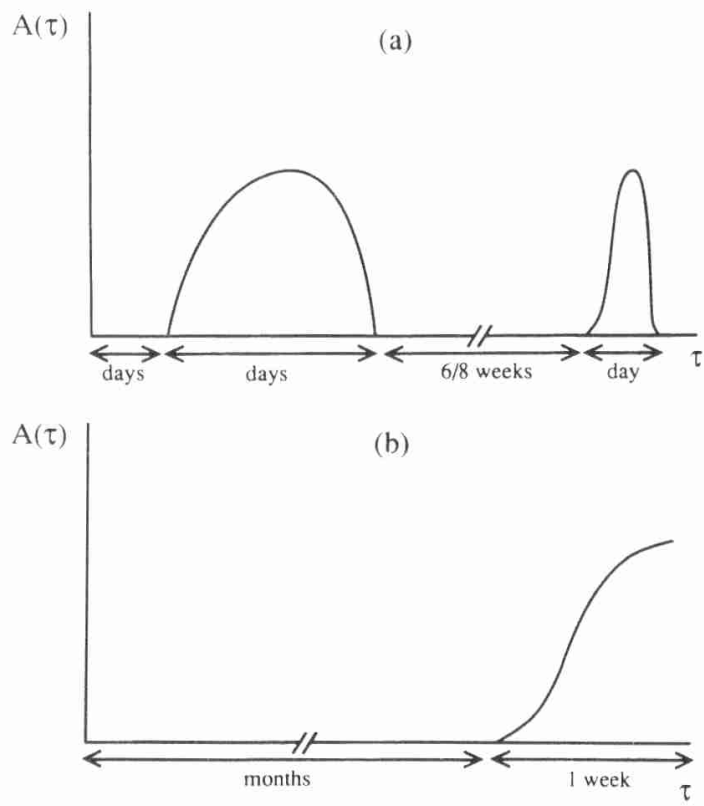

Figure 1. Examples of the shape of the function $\mathcal{A}(\tau)$ : (a) For a disease with a short infectious period followed by recrudescence, and (b) for a disease with a long pre-infectious period.

that are not yet infectious, $E$ stands for 'exposed'), the infective individuals $I$ (i.e. individuals that are infections) and the removed class $R$ (consisting in general of individuals that have been infected but are no longer infectious, and are not susceptible, where the interpretation of 'removed' can vary from (temporarily) immune to dead).

\subsection{Simple model}

For the simplest compartmental description of an epidemic caused by a microparasite we make the following assumptions: the population is closed (i.e. we assume that the time-scale of the epidemic processes of transmission and recovery is much shorter than the time-scale on which the population size changes due to natural births and deaths). This assumption will be removed when we consider the impact of host population dynamics in Section 3. Animals recover from the infection at a rate $\gamma$, after which they are immune to further infection; contacts between individuals are made at random; there is a constant transmission rate $\beta$. Consider a large population of constant size $N$, of which $S$ animals are susceptible to a given disease, $I$ are infective and $R$ animals have been removed. How many new cases arise per unit of time? The $I$ infectives make on average $\beta I$ potentially infective contacts per unit of 
time. Only a fraction $S / N$ of those contacts is with a susceptible. So, $\beta I S / N$ is the number of new cases arising per unit of time.

The above assumptions lead to the following system of differential equations for the susceptible, infective and removed classes

$$
\begin{aligned}
\frac{d S}{d t} & =-\frac{\beta}{N} S I \\
\frac{d I}{d t} & =\frac{\beta}{N} S I-\gamma I \\
\frac{d R}{d t} & =\gamma I .
\end{aligned}
$$

In Appendix $\mathrm{A}$ it is shown that the infectivity function that describes the same situation is given by $\mathcal{A}(\tau)=\beta e^{-\gamma \tau}$.

Compartmental models are usually named after the types of compartment that occur in progression. System (2.1) is therefore an example of an SIRmodel (if there were no recovery, i.e. if $\gamma=0$, one would speak of an SI-model, whereas with an added latency period it would be an SEI-model, etc.).

\subsection{Invasion}

The first question one could ask when studying the disease dynamics under the assumptions listed above, is whether or not an epidemic develops when a small number $I_{0}$ of infectives is introduced into a large population of size $S_{0}$ where everyone is susceptible $\left(S_{0}+I_{0}=N\right)$. This question is answered by the value of the basic reproduction ratio $R_{0}$, i.e. the expected number of secondary cases produced by one typical primary case during the entire period of infectivity in a population consisting only of susceptibles. Under the assumptions that lead to $(2.1)$ we have

$$
R_{0}=\frac{\beta}{\gamma}
$$

because on average one infective individual meets and infects $\beta$ susceptible individuals per unit of time, and it does this for a period of time of average length $1 / \gamma$. An epidemic can develop if $R_{0}>1$ (if each infected individual on average causes more than one new infection, a chain reaction will ensue), whereas the disease will disappear from the population without causing a chain reaction of new cases if $R_{0}<1$.

Given that $R_{0}>1$, the solutions to system (2.1) typically look like the curve in Figure 2. The number of infectives will at first rise steadily, will then reach a maximum value and decrease until it reaches zero and the infection disappears. The decrease can be understood by observing that the susceptible population becomes too small after a certain point in time for an infective individual to come into contact with susceptibles sufficiently often during the 


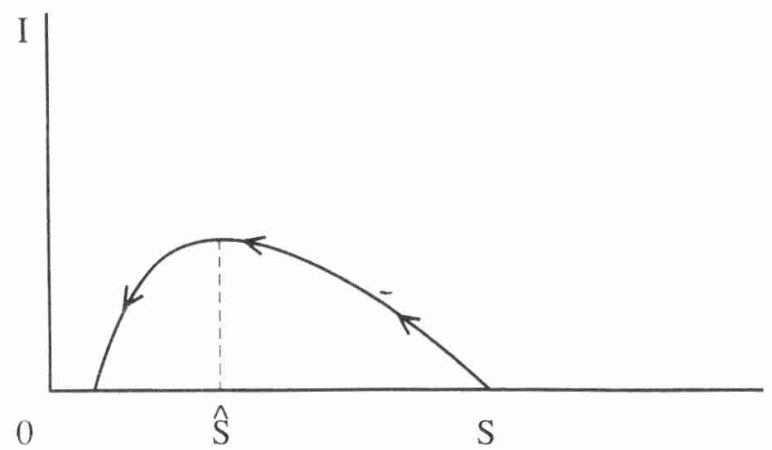

Figure 2. Typical solution trajectory for equation (2.1) in the $S, I$-plane. Note that $I$ achieves its maximum when $\hat{S}=S / N=\gamma / \beta$.

infectious period in order to cause new cases. Furthermore, the infectious period is of limited length, after which the individual becomes immune.

Remember that $R_{0}$ is defined in a virgin population, i.e. one consisting of susceptibles only. If $R_{0}>1$ and the epidemic progresses, then the susceptible population will decrease. In a non-virgin population, where a fraction $S / N<$ 1 is still susceptible, an infective individual will on average cause a number of new cases less than $R_{0}$ ( $R_{0}$ is an upper bound). One could use the term current (or effective, see Anderson (1982a), Busenberg and Cooke (1993)) reproduction ratio and denote it by $R_{c}(S)$, which depends on the currently available number of susceptibles, to describe the expected number of new cases per infective in a non-virgin changing population. Note that $R_{c}(S)$ is a function of time (because $S$ is), whereas $R_{0}$ is not. In the present case

$$
R_{c}(S)=\frac{S}{N} R_{0}=\frac{S \beta}{N \gamma}
$$

The epidemic reaches its maximum value (i.e. the maximum number of infectives present at the same time) at $d I / d t=0$. From the second equation of (2.1), we see that this occurs when the susceptible population has reached the value $\hat{S}=\gamma N / \beta$. This is equivalent to the value of $S$ where $R_{c}(S)=1$ (i.e. when, on average, each infective causes exactly one new infection in the non-virgin population). If $S<\hat{S}$, then $R_{c}(S)<1$ and the infection cannot persist in the population (we already intuitively described this above).

\section{$2.3 \quad$ Final size}

In this simple epidemic model, the size of the susceptible population will steadily decrease and approach a limit $S_{\infty}$. The question is whether $S_{\infty}=0$ (i.e. does the disease persist until every individual has suffered from it?). The 


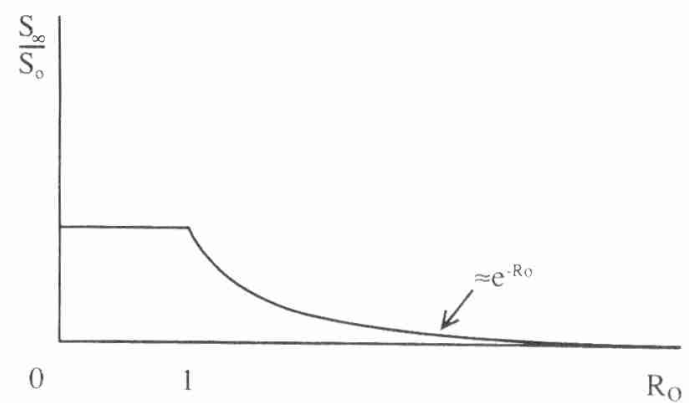

Figure 3. The ratio of the final size of the susceptible population to its initial size, for a simple epidemic model.

answer is already given by Kermack and McKendrick: no! More precisely, the following holds in good approximation (i.e. when $I_{0}$ is so small that $S_{0}+I_{0} \approx S_{0}$, for details see, e.g., Busenberg and Cooke (1993)):

- if $R_{0}>1$ then $S_{\infty}$ is found by solving the so-called 'final value' equation

$$
\ln \frac{S_{\infty}}{S_{0}}=R_{0}\left(\frac{S_{\infty}}{S_{0}^{\prime}}-1\right)
$$

In Figure 3, this relation is depicted graphically, and one can observe that the bigger $R_{0}$ is, the smaller $S_{\infty} / S_{0}$ will be. From the relation it follows that for large $R_{0}$ we have $\left(S_{\infty} / S_{0}\right)-1 \approx-1$, so $S_{\infty} \approx S_{0} e^{-R_{0}}$.

\subsection{Inflow of new susceptibles}

The behaviour of (2.1) is very simple: if an epidemic occurs $\left(R_{0}>1\right)$ then it will follow the general pattern of the curve in Figure 2 and we can calculate when it reaches its peak (when $R_{c}(S)=1$ ), and what the final size is. Of course, in (2.1) we focused on a very short time-scale, where the demographic changes that might occur in the meantime could be neglected. If we widen our view to a longer time-scale, then we have to take into account that fresh susceptibles join the population to replenish the susceptible 'pool'. The model would then become somewhat more complicated, and the dynamics of the solutions diversify. In Section 3, we review this in detail for compartmental models. Here we only give some general comments.

If we include inflow of new susceptibles (by births or migration) we can still retain the assumption that the time-scales of demographic and disease processes are different. In that case, the generic picture changes into that depicted in Figure 4. After the epidemic has run its course and the disease has died out, the susceptible population will again build up on the demographic time-scale, and after it has passed the critical level for $R_{c}(S)>1$, a new epidemic can occur if the disease is reintroduced. 


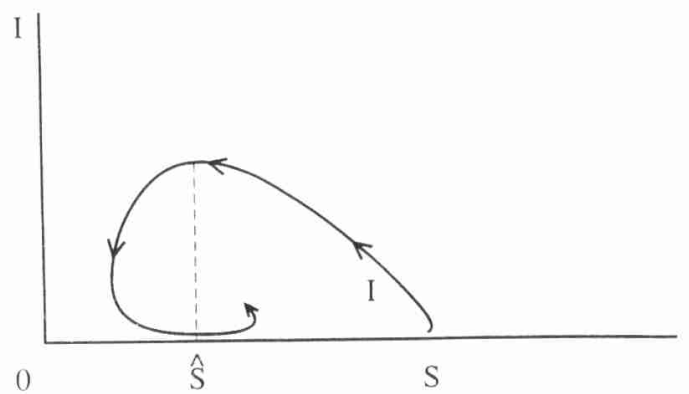

Figure 4. Typical solution trajectory in the S,I-plane for a simple epidemic model with birth or immigration of susceptibles, where the demographic timescale is long compared to the disease time-scale.

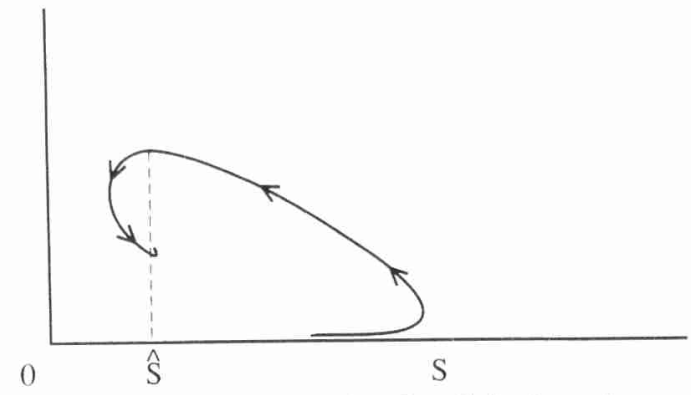

Figure 5. Typical solution trajectory in the $S, I$-plane for a simple epidemic model with birth or immigration of susceptibles, where the demographic and disease time-scales are similar.

If inflow of new susceptibles is important on the epidemic time-scale, then one can encounter various types of dynamic behaviour. One possibility is that a stable steady endemic state obtains, at precisely that level of the susceptible population where the corresponding $R_{c}(S)=1$ (when, on average, each case generates exactly one new case). This value of $S$ is often called the critical community size (see Bartlett (1960) and Schenzle and Dietz (1987)). If the susceptible population is above this size, then an infection can persist. This situation is depicted schematically in Figure 5. Alternatively, the system can show regular or irregular oscillations around an unstable endemic steady state. If no steady state exists, then the susceptible population can either show a steady increase, with the infected population increasing steadily as well (and a question can be whether the disease causes the population to grow at a slower rate than it would have done in absence of the disease), or the disease can die out. We discuss simple models in Section 3 that show this range of behaviour. 
As far as the disappearance of a disease from a population is concerned, it is useful to distinguish at least two types of stochastic aspects, linked to the recurrent epidemics and the endemic steady states described above (see Diekmann et al. (1995) for a more detailed discussion):

1. If population turnover is slow relative to disease transmission, we reach almost the final size situation of the closed epidemic before the gradual inflow of new susceptibles has any effect. In this case, there are very few infecteds and the size of the susceptible population is of the order of $S_{0} e^{-R}$, which is far below threshold. It will take a long time before the density is above threshold again, and during this period demographic stochasticity may easily lead to the extinction of the infectious agent. This is referred to as epidemic fade-out in Anderson and May (1991, p.20).

2. Even if the infective agent escapes extinction after the first outbreak and becomes established, there is still the possibility of extinction as a result of chance fluctuations. This is called endemic fade-out in Anderson and May (1991).

We end this section with some elementary remarks about control. If some control strategy (e.g. vaccination or culling) were being considered for a given endemic disease, then it would be interesting to evaluate its efficacy in eradicating the disease. Let us take vaccination in a homogeneous population as an example and consider the possibility for invasion in the partly vaccinated population. If a fraction $v$ of the susceptible population is made immune to infection at any given time, due to vaccination, then a fraction $(1-v)$ of the susceptibles remains available. If the vaccination strategy is to be successful, it has to bring the reproduction ratio $R_{v}$ in the vaccinated population below one. Note that $v=0$ (no vaccination) brings us back to the invasion problem in a virgin population. In the situation described by the model (2.1) we get $R_{v}=(1-v) \beta / \gamma$ as the expected number of secondary cases. For success we want $R_{v}<1$ which is equivalent to

$$
v>1-\frac{\gamma}{\beta}=1-\frac{1}{R_{0}}
$$

In Section 4, we discuss control strategy evaluation for more 'realistic' models.

\subsection{Density models}

In the literature one encounters models expressed not in absolute numbers of animals but in densities. Usually density refers to number of animals per unit area, but other density-measures may be important as well. For example, in 
the context of the larch-budmoth and its granulosis virus, Baltensweiler (see e.g. Baltensweiler (1968), Briggs et al. (this volume)) uses number of larvae per $7.5 \mathrm{~kg}$ of branches as a measure of density (see also Bowers et al. (1993)). For the rest of this section we will only consider spatial density.

If the area occupied by a population remains constant, then an increase in numbers is equivalent to an increase in density, and one might as well formulate the model in terms of absolute numbers as these are often intuitively easier to grasp. If the population expands the area it occupies when numbers increase, then the density could stay roughly constant and it depends on the type of problem one is studying whether the sizes of the various classes are better expressed in numbers than in densities. For example, when studying effects of the environment on host population dynamics (limits in growth) one might opt for densities (see however Section 3.3).

The main reason for the use of densities is the immediate connection to the law of mass-action from chemical reaction kinetics which underlies the transmission term in many epidemic models. The mass-action assumption originally states that the rate at which molecules of types $A$ and $B$ collide (i.e. the number of collisions per unit volume per unit of time) in a wellstirred reaction vessel, is proportional to the product of the concentrations of $A$ and $B$. Translated into susceptible and infective individuals making pairwise contacts in a homogeneously mixing population, the assumption reads that the number of contacts per unit area per unit of time is proportional to the product of the density of susceptibles $s$ and the density of infectives $i$. The main assumption, apart from homogeneity, underlying this is that locally the probability per unit of time of a contact between two individuals is the same for any pair. In the situation of model (2.1) this leads to a transmission term of the form $\hat{\beta}$ si, where $\hat{\beta}$ is the transmission rate constant. In De Jong et al. (1995), both theoretical and experimental results are discussed that show that the correct counterpart of mass-action if one expresses class-sizes in absolute numbers is given by transmission terms of the form: $\beta S I / N$, as in (2.1).

In Dwyer and Elkington (1993), a model for a nuclear polyhedrosis virus in Gypsy moths is discussed where the description in densities is particularly apt (Briggs et al., this volume). One of the variables is the quantification of infectious virus-particles in the environment which can of course only be expressed as a density (here number per unit volume) because absolute numbers are, as in chemical reactions, not appropriate.

\section{Adding various complexities}

\subsection{The basic SI-model}

In the present section we will add more biological complexity ('realism') to the simple model (2.1) and describe the dynamic behaviour of the models thus 


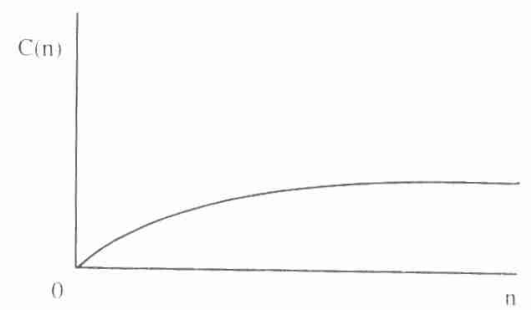

Figure 6. The contact rate $C$ as a function of population density $n$.

obtained. In this subsection we add a fow basic details, in further subsections we then look at various extensions with examples and applications. We start however, by disregarding recovery (i.e. take $\gamma=0$ ), and defer study of the influence of an 'R-class' to Section 3.6. For a detailed review and analysis of SI-models, including many of the extensions given below, see Zhou and Hethcote (1993).

In deference to its potential importance for wildlife diseases, we first of all relax the requirement that the population size $N$ is constant. Furthermore, let each individual make contact with $C(N)$ other individuals per unit of time. The definition of contact depends upon the mode of transmission of the disease under study. Given that the contact is between an infective and a susceptible individual, the transmission rate is $\beta C(N)$. For each contact the probability that the 'contactee' is susceptible is $5 / N$ (we assume homogeneous mixing), and hence the total number of infectious contacts per unit time is $\beta C(N) I S / N$. The expression $\beta C(N) I / N$ is often referred to as the force of infection. (Anderson and May 1991), being the fraction of the susceptible population that the infectives are able to contact and infect per unit of time.

The opportunity for contact depends upon the local population density. Heesterbeek and Metz (1993) have shown that, when it is taken into account that contacts have a non-negligible average duration, the relationship between the contact rate $C$ and the (spatial) population density $n$ in a homogeneously mixing population is as shown in Figure 6. The density itself may change as a function of population size $N$. If we consider a wild animal population then at low numbers the area occupied will not change initially as the number of animals slowly increases (i.e. density will increase linearly with population size). After some critical density has been reached animals will disperse or widen the territory occupied and density will temporarily drop only to increase again as numbers rise further to take up the enlarging area. As a caricature one could picture the relationship as shown in Figure 7 (dotted line) and approximate the general trend by a saturating curve (solid line). Combining the 'shapes' of Figures 6 and 7, one can easily show that $C$ as a function of $N$ is as in Figure 8 , which is similar to the form $N /\left(c_{1}+c_{2} N\right)$ postulated by Dietz (1982). 


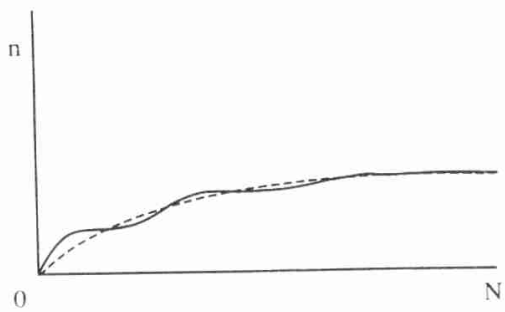

Figure 7. Population density $n$ as a 'heuristic' function of population size $N$.

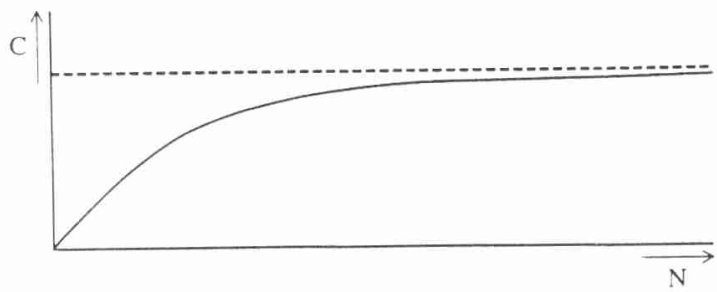

Figure 8. The contact rate $C$ as a function of population size $N$. Reproduced from Roberts and Heesterbeek (1993), with permission.

A more careful argument along similar lines, see Diekmann et al. (1994), results in a counterintuitive $S$-shaped relation between population size and contact rate. This might prove to be the correct relation certainly for animals that display territorial behaviour or have specific contact structures like the seal population they discuss. There is a clear need for more detailed submodels of the contact process.

We now add rudimentary demographics of the animal population to the basic model, and include a general contact rate function $C(N)$. As pointed out by Anderson and May (1979), a central question here is: can the parasite regulate the size of the host population? The only way to leave the infected class $I$ is by death, either due to the disease or from natural causes. Let the (constant) per capita birth and death rates in the absence of disease be $a$ and $b$ respectively, and assume $a>b$ (hence the population increases exponentially in the absence of disease). The model (2.1) becomes

$$
\begin{aligned}
& \frac{d S}{d t}=a N-b S-\beta \frac{C(N)}{N} S I \\
& \frac{d I}{d t}=\beta \frac{C(N)}{N} S I-(\alpha+b) I,
\end{aligned}
$$

where $\alpha$ is the increase in host mortality due to disease. It is often more convenient to work in terms of the total population size $N$ and the number of infectives I. Adding (3.1) and (3.2) (and copying (3.2) with $S=N-I$ ) we obtain

$$
\frac{d N}{d t}=(a-b) N-\alpha I
$$




$$
\frac{d I}{d t}=\beta \frac{C(N)}{N}(N-I) I-(\alpha+b) I .
$$

System (3.3) has steady state solutions defined by $(N, I)=(0,0)$ and $\left(N^{*}, I^{*}\right)$ with

$$
\begin{aligned}
I^{*} & =\frac{a-b}{\alpha} N^{*} \\
C\left(N^{*}\right) & =\frac{\alpha(\alpha+b)}{\beta(\alpha+b-a)} .
\end{aligned}
$$

As equation (3.5) does not explicitly define $N^{*}$, an obvious requirement for the steady state to exist is that (3.5) has a solution. This is not a problem if, for example, $C(N)$ is as in Figure 8 , with the limit for large $N$ sufficiently large (i.e. larger than the right-hand side of (3.5)). Most authors proscribe that $C(N)$ must be an increasing function of $N$, with some technical requirement to ensure that $N^{*}$ is defined uniquely (see, e.g., Thieme (1992) and the references given there). If ( 3.5$)$ indeed has a unique solution, then a positive steady state exists, and the disease can regulate the population when $\alpha>a-b$. This requirement may be interpreted as regulation being achieved if the diseaseinduced mortality rate exceeds the intrinsic growth rate of the population. In this context, regulation means that a population that is increasing in size in the absence of the disease, is limited to a steady size in the presence of the disease (Anderson and May 1979). Dickmann and Kretzschmar (1991) proved that the steady state defined by (3.4-5), if it exists, is globally asymptotically stable, i.e. solutions will always tend to that steady state over time.

A related question is what happens to the dynamics of (3.3) if the steady state (3.4-5) does not necessarily exist. From (3.3b) it is apparent that if $I$ is very small, then $d I / d t<0$ whenever $\beta C(N)<\alpha+b$. Diekmann and Kretzschmar (1991) investigated the special case

$$
C(N)=\frac{N}{c+N}
$$

in detail. Formula (3.6) has the general shape given in Figure 8. The dynamics of solutions of (3.3) may, with this choice for $C(N)$, be summarised as follows, in terms of the value of the transmission rate constant $\beta$ in relation to the other parameters:

$$
\begin{array}{ll}
\text { (i) } 0 \leq \beta<b+\alpha & N \rightarrow \infty, I \rightarrow 0 \\
\text { (ii) } b+\alpha<\beta<a+\alpha & N \rightarrow \infty, I \rightarrow \infty \\
\text { (iii) } a+\alpha<\beta<\ell & I / N \rightarrow 0 \\
& N \rightarrow \infty, I \rightarrow \infty \\
\text { (iv) } \beta>\ell:=\alpha(\alpha+b) /(\alpha+b-a) & I / N \rightarrow(\beta-\alpha-a) /(\beta-\alpha)>0 \\
& N \rightarrow d /(\beta-\ell), \\
& I / N \rightarrow(a-b) / \alpha .
\end{array}
$$



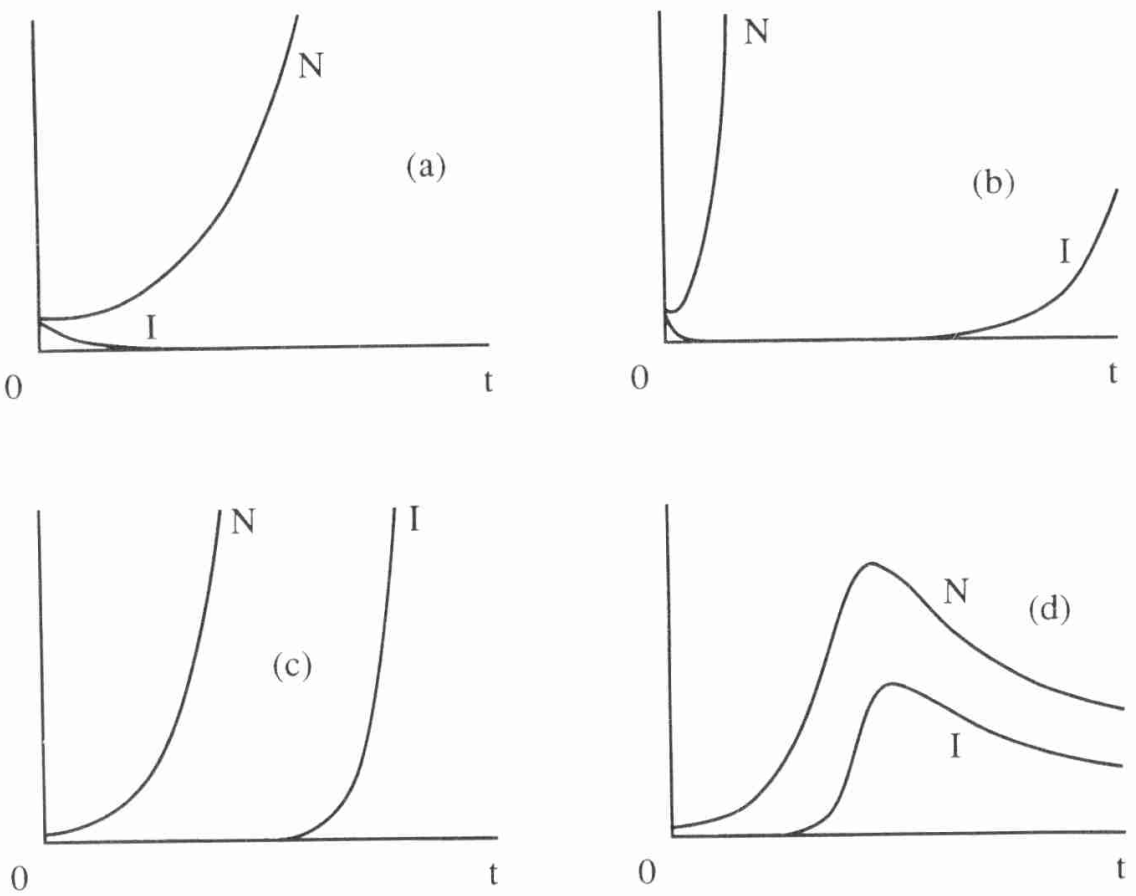

Figure 9. Numerical solutions of equation (3.3) with $a=\alpha=2, b=$ $1, C(N)=N /(5+N)$ and a) $\beta=2$; b) $\beta=3.5$; c) $\beta=5$ and d) $\beta=8$; satifying conditions (i)-(iv) above, respectively. Reproduced from Roberts and Heesterbeek (1993), with permission.

Inspection of the proofs in Diekmann and Kretzschmar (1991) makes it clear that similar results hold true if $C(N)$ is any increasing function. In words, the four possibilities are, for increasing values of the transmission rate $\beta$ : i) the disease becomes extinct and the host population grows without bound; ii) both the susceptible and infective populations grow without bound, but the proportion that are infective tends to zero; iii) both the susceptible and infective populations grow without bound, but the proportion that are infective tends to a positive finite limit; and iv) both the susceptible and infective populations tend to a bounded steady state ((3.4-5)). The four possibilities are illustrated schematically in Figure 9.

If the contact rate $C(N)$ is a constant for all $N$, then equation (3.5) can only be solved if the constant happens to be equal to the right-hand side of 
(3.5). For this case we have the same possibilities (i)-(iv) from the previous paragraph, but the bounded steady state of (iv) is now the steady state $(N, I)=(0,0)$ (see Busenberg and Van den Driessche 1990).

\subsection{Disease-dependent host demographics}

In many cases diseases will reduce the fertility of the host animal (for an excellent biological discussion see (iulland, this volume). Examples are fungal infection of geese (Smith and Dobson 1992), brucellosis causing abortions in elk (Peterson et al. 1991) and microsporidian infection of flour beetles (Onstad and Maddox 1990). See Dobson and Hudson (this volume) for additional evidence from data of reduced host fitness and reduction to lower population densities in the presence of pathogens (for microparasites from three different biological classes). Briggs of al. (this volume) provide an extensive discussion of the relationship between disease and host demography for microparasites of insects.

In Diekmann and Kretzschmar (1991) this effect on the birth-term is modelled by including a rudimentary description of pair formation. A pair can be formed between two susceptibles, a susceptible and an infective (in which case the rate of producing offspring is reduced by a factor $\xi$ ) or two infectives (reduction by factor $\xi^{2}$ ). This leads to

$$
\frac{d N}{d t}=a \frac{(N-(1-\xi) I)^{2}}{N}-b N-\alpha I .
$$

The system (3.31, 3.6,3.7) was analysed by Diekmann and Kretzschmar (1991), who determined the following dynamic behaviour. There exist two threshold parameters $\xi_{T}$ and $\xi_{P}$. If $\xi>\xi_{T}$ then the behaviour of solutions is qualitatively the same as that of solutions to $(3.3,3.6)$ (which corresponds to $\xi=1)$. If $\xi_{P}<\xi<\xi_{T}$, there exists a range of the parameter $\beta$ for which there is bistablility (long term behaviour dependent on initial conditions). If $0<\xi<\xi_{P}$ there exists a range values of $\beta$ for which stable periodic solutions occur.

In Zhou and Hethote (1993) reduction of fertility is also studied, but with a birth-term which is not quadratic. In this case no cyclic behaviour is possible.

As an extension of (3.7), it may be interesting to study the following variant

$$
\frac{d N}{d t}=a \frac{(N-(1-\xi) I)(N-(1-\eta) I)}{N}-b N-\alpha I
$$

which could mimic different effects of the disease on the fertility of males and females (Hethcote, personal communication), or different effects on female fertility depending on whether a susceptible female contacts an infective male or whether the female is itself infected. 


\subsection{Host population dynamics}

Host populations do not grow in an unbounded manner. In reality the birth and/or death rate will depend on the host population density, birth rate being a non-increasing function of density and death rate non-decreasing. The derivation in the previous subsections was in terms of size rather than density, but as in the argument for the contact rate function in 3.1 , there is a direct correspondance between the two. We will continue to use population size as a variable. Consider the equation

$$
\frac{d N}{d t}=(A(N)-B(N)) N-\alpha I
$$

where $A$ and $B$ are birth and death rates, respectively, as functions of $N$ with $d A / d N \leq 0$ and $d B / d N \geq 0$. Equation (3.8) is the analogue of (3.3a), for density dependent population growth. The term in brackets ensures that in the absence of disease the population size tends over time to the carrying capacity $K$, where $A(K)=B(K)$, with positive growth if $N<K$, and negative growth if $N>K$. Often logistic population regulation is assumed with $A(N)-B(N)=r(1-N / K)$ (where $r=a-b)$. Nonlinear effects in population growth are in response to the local population density, and an examination of the community structure is necessary to ensure that any model is correctly defined. For models with a constant rate of recruitment (i.e. $\mathrm{N}$-independent) or immigration into the population, see Zhou and Hethcote (1993) and the references given there. Of course, wild animal populations do not, in general, breed continuously throughout the year, but have a seasonal pattern. These periodic birth patterns could be modelled by taking $A(N)=$ $a(1+t \phi(t)) N$, where $\phi$ is a periodic function of time. We will not discuss this complication in more detail but refer to Roberts et al. (this volume), Grenfell (1992), Bolker and Grenfell (1993) and the references given there.

Equation (3.3b) becomes

$$
\frac{d I}{d t}=\beta \frac{C(N)}{N}(N-I) I-(\alpha+B(N)) I .
$$

The above system (3.8-9) has three steady state solutions: the trivial one $(N, I)=(0,0)$ which is unstable, the disease-free steady state $(N, I)=(K, 0)$, and the endemic steady state $\left(N^{*}, I^{*}\right)$ which only exists if the basic reproduction ratio

$$
R_{0}=\frac{\beta C(K)}{\alpha+B(K)}
$$

is greater than one. The solution dynamics of (3.8-9) may be summarised as follows. If $R_{0}<1$, the disease cannot persist, and all solutions tend to the disease-free steady state $(K, 0)$. If $R_{0}>1$, the disease persists in the population, and solutions tend to the endemic steady state $\left(N^{*}, I^{*}\right)$, where 


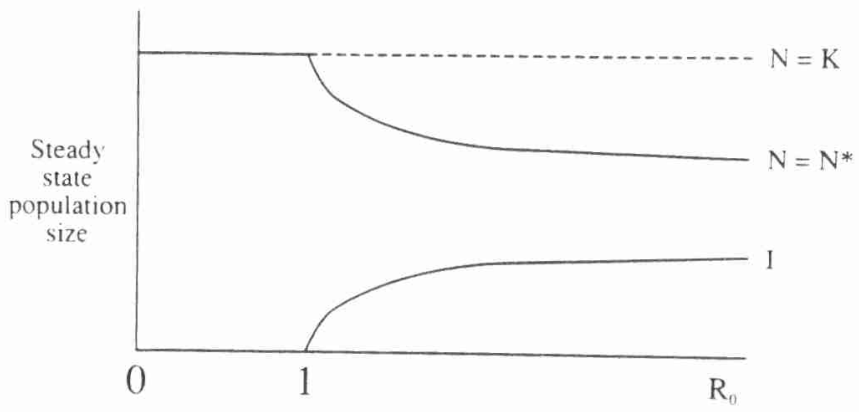

Figure 10. Steady state population size $N$ and the size of the infectious population $I$ as functions of $R_{0}$ for equations (3.8-9). The broken line signifies an unstable steady state. Reproduced from Roberts and Heesterbeek (1993), with permission.

$N^{*}<K$. The steady state value $N^{*}$ as a function of $R_{0}$ is given in Figure 10 .

As $R_{0}$ depends on $K$ an alternative way of expressing the threshold theorem is to define $K_{T}$ as that carrying capacity for which $R_{0}=1$. The threshold for disease persistence can then be expressed as $K>K_{T}$, where

$$
\frac{\beta C\left(K_{T}\right)}{\alpha+B\left(K_{T}\right)}=1 .
$$

The validity of this as a threshold, or indeed the existence of a unique solution for $K_{T}$, depends upon the precise form of the functions $B(N)$ and $C(N)$. For example, for the extreme case $C(N)=N$, with $B(N)=b$, constant, $K_{T}=K / R_{0}$ with $R_{0}=\beta K /(\alpha+b)$. For the other extreme $C(N)$ a constant $(=1), R_{0}=\beta /(\alpha+b)$ (compare Section 2). For the latter case, both the contact rate $C$ and $R_{0}$ are independent of population size, and the concept of $K_{T}$ is meaningless. In Bouma et al. (in preparation) and in De Jong et al. (1995), both theoretical arguments and experimental evidence are discussed that show that $R_{0}$ does not depend on population size at constant population density.

\subsection{Vertical transmission}

Vertical transmission of a parasite occurs when it is passed from mother to offspring either before or at birth. Examples include venereal spirochaetosis of rabbits (transmission at birth) (Smith and Dobson 1992) and insect baculoviruses (see Briggs et al. (this volume) for a review). Pseudo-vertical transmission is the term used when the disease is passed from mother to offspring after birth, due to unusually close contact. An example of this is 
tuberculosis in possums (Roberts 1992), where the young are infected while being carried in the pouch. For both cases the mathematics are essentially the same. Equation (3.9) is modified by a term specifying the addition to the infective class due to births to infective parents

$$
\frac{d I}{d t}=p A(N) I+\beta \frac{C(N)}{N}(N-I) I-(\alpha+B(N)) I .
$$

A complete analysis of the SI-model with vertical transmission for constant births and deaths, for $C(N)=N$, and with density-dependent (logistic) host death can be found in Busenberg and Cooke (1993).

\subsection{The effect of long latency periods}

Many diseases have a long latency period before the individual becomes infective. This may be modelled by introducing the $E$-class, $N=S+E+I$, see Section 2. Equation (3.9) is replaced with separate equations for the $E$ and $I$ classes,

$$
\begin{aligned}
\frac{d E}{d t} & =\beta \frac{C(N)}{N}(N-E-I) I-(\sigma+B(N)) E \\
\frac{d I}{d t} & =\sigma E-(\alpha+B(N)) I
\end{aligned}
$$

where $\sigma$ is the rate at which infected animals become infective (so, the duration of the latency period is assumed to be exponentially distributed with average value $1 / \sigma)$. As explained at the end of Appendix $\mathrm{A}, R_{0}$ would not be affected by an $E$-class if no deaths occured in this class. In the present model, deaths do occur and this factor has to be taken into account. Only a fraction $\sigma /(\sigma+B(K))$ of the latent individuals is expected to survive the latency period in a virgin population, so

$$
R_{0}=\frac{\sigma \beta C(K)}{(\sigma+B(\Lambda))(\alpha+B(K))} .
$$

Rather than present an analysis of equations $(3.8,3.11-12)$ in the abstract, we proceed to discuss a specific example.

\section{Example: Rabies in foxes}

A model for the dynamics of rabies in European foxes discussed by Anderson et al. (1981) featured a density dependent death rate regulating the host population in the absence of disease, a constant birth rate, but only susceptible foxes giving birth, and contact rate function $C(N)=N$. In our notation the equations are

$$
\frac{d N}{d t}=r\left(1-\frac{N}{K}\right) N-a(E+I)-\alpha I
$$




$$
\begin{aligned}
\frac{d E}{d t} & =\beta(N-E-I) I-\left(\sigma+b+r \frac{N}{K}\right) E \\
\frac{d I}{d t} & =\sigma E-\left(\alpha+b+r \frac{N}{K}\right) I .
\end{aligned}
$$

This model has been extensively analysed by Anderson (1982a). The condition for persistence of the disease is

$$
R_{0}=\frac{\sigma \beta K}{(\sigma+a)(\alpha+a)}>1 \text { or } \quad K>K_{T}=\frac{(\sigma+a)(\alpha+a)}{\sigma \beta K} .
$$

The appearance of $a$ instead of a death rate in the denominator of the expression for $R_{0}$ seems surprising at first, but when $N=K$ (at carrying capacity), births and deaths balance (i.e. $a=r+b K / K$ ).

However, the regulated population state may be a constant value or a stable cycle, depending upon the parameter values. If there is a constant steady state solution trajectories exhibit damped oscillations as they tend towards it, and for typical parameter values the damping period is long (3-4 years). Anderson (1982a) showed that stable cycles would occur if both the fox carrying capacity $K$ and the mean latency period $1 / \sigma$ were high. The stability of the limit cycles has been confirmed analytically by Swart (1989). If the latency period is short cycles do not occur. As we saw earlier, cyclic behaviour is impossible in the SI-model that is obtained from Anderson's model in the limit $\sigma \rightarrow \infty$ or $1 / \sigma \rightarrow 0$. This is therefore a situation where a delay in transmission caused by a long latency period can destabilise the steady state of a system, and cause the population size and the number of diseased animals to exhibit periodic cycles (see also Bolker et al., Dye et al. both this volume).

We see that there are at least two ways in which to get cyclic behaviour if one starts off with the basic SI-model: one is to introduce a time-delay, as outlined above, the other is to introduce special nonlinear feedback in the birth-term, as outlined in Section 3.2. For recent reviews of periodic behaviour in epidemic models, both compartmental and more general, see Hethcote and Levin (1989) and Liu (1992).

\subsection{The effect of acquired immunity}

Until now in this section, we have assumed that once an infection is acquired there is no recovery. This is not the case for many infections, and in some cases, the host animal is immune to contracting the infection again for some period after its recovery, or indeed for life. It is common to model this by the introduction of an $R$ (removed) class (see Section 2). For our simple model, equation (3.8) remains unchanged, and equation (3.9) becomes

$$
\frac{d I}{d t}=\beta \frac{C(N)}{N}(N-I-R) I-(\alpha+B(N)+\gamma) I
$$


where $\gamma$ is the probability per unit of time of recovery from the disease and $N=S+I+R$. The dynamics of the $R$-class is expressed by

$$
\frac{d R}{d t}=\gamma I-(B(N)+\rho) R
$$

where $\rho$ is the probability per unit of time of loosing immunity (upon which the individuals re-enter the susceptible class with rate $\rho R$ ). The system (3.8.3.13-14) has, apart from the trivial steady state, a nontrivial diseasefree steady state $N=S=K, I=R=0$, and an endemic steady state exists when $R_{0}>1$, where

$$
R_{0}=\frac{\beta C(K)}{\alpha+B(K)+\gamma} .
$$

For a detailed analysis of SIRS-models with $A(N)=a$ and $B(N)=b$, constants, see Thieme (1992). Greenhalgh and Das (1993) do a similar analysis with a density dependent death rate (logistic deaths). Both papers review most of the literature on this problem. See also Anderson and May (1991), Hethcote (1994).

Examples of diseases where a removed class plays a role in the dynamics, include avian pox virus in hawks (Graham and Halliwell 1986) and pigeon pox in (wild) pigeons (Cubb 1986). In the former case, the host is permanently immune after recovery, in the latter it becomes susceptible again after at least one year.

\section{Control aspects}

A model is primarily a means by which the dynamics of the infection may be understood, but the motivation for the construction of the model is often to aid in the planning of control. The type of control measure that is proposed will depend on the desired end result in terms of both the disease prevalence and the population density. For example, if a wild animal population is acting as a reservoir for a disease of humans or domestic livestock, then killing animals on a regular basis may be the most appropriate way of decreasing the host population size, and hence the amount of disease present. Alternatively, if the reason for control is that the disease is present in a population that is being conserved, then killing animals is not a suitable control measure, and vaccination may be considered. Vaccination will, however, reduce host mortality and may increase the population above desired levels. In this section models for a variety of different strategies for the control of hosts and/or pathogens are discussed. See also Anderson (1982a,b). 


\subsection{Culling}

Consider a program with a fixed per capita culling rate $\delta$. Equations (3.8-9) become

$$
\begin{aligned}
\frac{d N}{d t} & =(A(N)-B(N)) N-\delta N-\alpha I \\
\frac{d I}{d t} & =\beta \frac{C(N)}{N}(N-I) I-(\alpha+B(N)+\delta) I
\end{aligned}
$$

and $R_{\delta}$, the basic reproduction ratio in the presence of control, is defined by

$$
R_{\delta}=\frac{\beta C\left(K_{\delta}\right)}{\alpha+B\left(K_{\delta}\right)+\delta}
$$

where $A\left(K_{\delta}\right)-B\left(K_{\delta}\right)-\delta=0$ (the expression reduces to $R_{0}$ from subsection 3.3 when $\delta=0)$. Hence, regular culling of host animals may eradicate the disease from the population if

$$
\delta>\beta C\left(K_{\delta}\right)-\left(\alpha+B\left(K_{\delta}\right)\right)
$$

which would reduce the size of the remaining disease-free population to $K_{\delta}<$ $K$. Similar models where culling has been investigated as a method of reducing the prevalence of a wildlife disease include tuberculosis in possums (Roberts 1992), rabies in foxes (Anderson 1982a), rabies in raccoons (Coyne et al. 1989), and tuberculosis in badgers (Anderson and Trewhella 1985). See also Dobson and Hudson (this volume).

When only infected animals are killed upon discovery, this effectively means an increase in the differential mortality rate $\alpha$. A successful example of this type of control concerns dourine (Trypanosoma equiperdum) in wild horses and zebra (Smith and Dobson (1992), see also Section 5).

\subsection{Vaccination}

Vaccination of a wild animal population may, for example, be achieved by the spreading of baits containing an oral vaccine. The effects of this control measure may be modelled by introducing a new class, $V$, for animals that are immune to infection due to vaccination. For our simple model, equation (3.8) remains unchanged and in (3.9), N-I is replaced by $N-I-V$, because now $N=S+I+V$. If we introduce a per capita vaccination rate $q$, then

$$
\frac{d V}{d t}=q(N-I-V)-(B(N)+\nu) V
$$

where it has been assumed that vaccination has no effect on animals in the $I$-class, and where $\nu$ is the rate of loosing the protected status due to, for 
example, loss of memory in the immune system of the host. The basic reproduction ratio $R_{q}$ under vaccination is given by

$$
R_{q}=\frac{\beta C(K)(B(K)+\nu)}{(\alpha+B(K))(B(K)+\nu+q)} .
$$

Hence, regular vaccination of host animals may eradicate the disease from the population if

$$
q>(B(K)+\nu)\left(R_{0}-1\right)
$$

which would increase the size of the population to $N=K$, with a proportion $v=V / N=q /(B(K)+v+q)$ of these immune due to vaccination. If we do not vaccinate at a rate $q$, but vaccinate in such a way that at any given time a fraction $v$, as defined above, is vaccinated, we find from (4.1) and (4.2) that the criterion for success is

$$
v>1-\frac{1}{R_{0}},
$$

an equality we derived for the simple model (2.1) in Section 2.4 (with a different $R_{0}$ of course).

Examples of vaccination strategies include, rabies in foxes (Anderson 1982b), rabies in raccoons (Linhart et al. 1991), and brucellosis in elk (Peterson ot al. 1991). See also Dobson and Hudson (this volume). The strategy may be to spread baits containing an oral vaccine (for example to control rabies in foxes), or to capture animals, vaccinate and release them (for example seals with phocine distemper virus; Grenfell et al. (1992)).

\subsection{Chemotherapy}

The effect of mass chemotherapy to control disease may be modelled in a similar way to that of culling and vaccination discussed above. Equation (3.8) remains unchanged, but if infected animals are successfully treated with a per capita rate $h$, then equation (3.9) is replaced by

$$
\frac{d I}{d t}=\beta \frac{C(N)}{N}(N-I) I-(\alpha+B(N)+h) I .
$$

It is easily seen that the basic reproduction ratio $R_{h}$ of the disease under chemotherapy is

$$
R_{h}=\frac{\beta C(K)}{\alpha+B(K)+h}
$$

and chemotherapy is successful if

$$
h>(\alpha+B(K))\left(R_{0}-1\right) .
$$

Note that the effect of introducing chemotherapy into the model is the same as the effect of increasing the rate at which the host animal recovers from the disease. 


\subsection{Comparison of control strategies}

The similarity between equations (4.2) and (4.3) suggests that the efficacy of different strategies could be compared. In fact, the ratio of the proportion of hosts that must be vaccinated annually to the proportion that must be treated by chemotherapy is $q / h=(\nu+B(K)) /(\alpha+B(K))$. Hence, if $\nu>\alpha$ the rate of vaccination required to achieve disease eradication is higher than the rate of treatment required for the same purpose. If the majority of the cost is due to the logistics of the operation, rather than the chemical agents employed, then this formula provides a simple means of comparing the two options on a financial basis.

It is more difficult to compare the effects of culling and vaccination or chemotherapy, as this option establishes a new population size $K_{\delta}<K$, which may reduce both the host mortality rate $B(N)$ and the contact rate $C(N)$. If the host species is regarded as a pest in its own right this could be desirable (for example possums, rabbits, foxes), but as we mentioned in the introduction to Section 4, a culling strategy would defeat the purpose of a disease control operation that was part of a wildlife conservation plan. A comparison of relative rates of culling and the other two strategies is dependent on the precise forms of the functions $B(N)$ and $C(N)$, and will therefore differ between diseases and host demographic situations.

\section{Heterogeneity}

If we add more realism to the basic models of Section 3, by taking into account the fact that not all individuals are the same, matters quickly become much more complicated. There are many types of heterogeneity that could have important influences on susceptibility, infectivity and the structure of the contact process (who meets whom how often). Important types are age (in sexually transmitted diseases, viral diseases, maybe always important), spatial location (for example with respect to rabies if one is interested in spatial spread, see Mollison and Levin (this volume), gender (influences on behaviour, for example in sexually transmitted diseases), social structure (for example families, home ranges), different host species in the life cycle of the parasite (for example vector transmitted diseases, see Dye (this volume)), genetic diversity (see Lively and Apanius (this volume)) and others.

Heterogeneous characteristics may be qualitative (e.g. male/female), quantitative and discrete (e.g. number of offspring) or continuous (e.g. age). Most results on the dynamic behaviour of heterogeneous models are for the case where a finite number of different subgroups of individuals is recognised. Analysis of infinite dimensional heterogeneous models is predominantly aimed at age-structure and spatial structure (e.g. characterising rates of spatial spread of diseases, see the review by Metz and Van den Bosch (1995)). We 
will not go into details here about the dynamic properties and formulation of heterogeneous models in general, for recent guides to the literature see Anderson and May (1991) and Diekmann et al. (1995).

For this paper we restrict ourselves to a specific two-dimensional example of a sexually transmitted disease. Sexually transmitted diseases (STD's) of animals, including wildlife (such as herpes in baboons, STD's of rabbits, crabs, geese, and Chlamydia infections of the koala) have recently been reviewed in Smith and Dobson (1992). For illustrative purposes we give below a simple model for the STD dourine of wild horses and zebra. This example captures some of the essentials of the host-pathogen system. We recognise two types of animal in the population, females (mares, 1 ) and males (stallions, 2) and take into account the following: transmission rates differ for male to female and female to male; there is only heterosexual transmission; the disease is fatal in males whereas females often recover to become susceptible again. We can model this situation using an extension of system (2.1) to include differential mortality $\alpha_{i}$ (i.e. mortality due to the disease) and recovery without immunity $\rho_{i}, i \in\{1,2\}$ (we take the removal rate $\gamma_{1}=\gamma_{2}=0$, because immunity is generally absent in STD infections). We obtain at first the more general system

$$
\begin{aligned}
\frac{d S_{i}}{d t} & =a-\beta_{i 1} I_{1} \frac{S_{i}}{N_{i}}-\beta_{i 2} I_{2} \frac{S_{i}}{N_{i}}+\rho_{i} I_{i}-b S_{i} \\
\frac{d I_{i}}{d t} & =\beta_{i 1} I_{1} \frac{S_{i}}{N_{i}}+\beta_{i 2} I_{2} \frac{S_{i}}{N_{i}}-\rho_{i} I_{i}-\alpha_{i} I_{i}-b I_{i}
\end{aligned}
$$

for $i \in\{1,2\}$, with $N_{i}=S_{i}+I_{i}$, where we have assumed a constant inflow of susceptibles into the population and a per capita death rate $b$. Under our assumptions for dourine we can assume $\beta_{11}=\beta_{22}=0$ (no homosexual transmission), $\rho_{2}=0$ (neglect recovery for males), $\alpha_{1}=0$ (neglect diseaseinduced death of females), and $\beta_{12} \neq \beta_{21}$. Model (5.1) is an example of an SIS-model, frequently encountered in the study of human STD's (see, e.g. Hethcote and Yorke 1984). Models like (5.1) with various complications added and with obvious generalisation to an arbitrary number of $n$ types of individual have been analysed extensively over the last years as a direct result of the HIV-epidemic in humans (see e.g. Hethcote and Van Ark 1992). See also Begon and Bowers (this volume), where multispecies models with a similar structure are discussed. Criteria for the existence and stability of steady states have been given for many cases and the area is very much one, to use Hethcote's words, of 1001 variations on the same theme (Hethcote 1994).

In Appendix B it is shown that the basic reproduction ratio for the situation described by (5.1), simplified by the assumptions on the parameters 
below it, is given by

$$
R_{0}=\sqrt{\frac{\beta_{21}}{\alpha_{2}} \frac{\beta_{12}}{\rho_{1}}} .
$$

If we study the effect of killing infected animals that are discovered, we could mimic this control strategy by increasing $\alpha_{1}$ and $\alpha_{2}$ by an amount $k$ which could represent the rate at which infected animals are detected (with an available diagnostic test on capturing). The reproduction ratio $R_{k}$ is then given by

$$
R_{k}=\sqrt{\frac{\beta_{21}}{\alpha_{2}+k} \frac{\beta_{12}}{\rho_{1}+k}},
$$

leading to a criterion for success

$$
k>\frac{1}{2}\left(\sqrt{\left(\rho_{1}+\alpha_{2}\right)^{2}+\alpha_{2} \rho_{1} R_{0}^{2}}-\left(\rho_{1}+\alpha_{2}\right)\right) .
$$

Of course, for horse-herds living in captivity, the detection rate $k$ will be much higher than in the wild. This control method for T. equiperdum has been applied successfully in various parts of the world (Smith and Dobson 1992).

\section{Conclusion}

This chapter presents a range of mathematical tools which can be used to explore the rich and increasingly well-documented dynamics of microparasitic diseases in wildlife host populations. Starting from the basic mathematical background, we have tried to show that the resulting models can be used to address fundamental epidemiological questions (how do we model the infection process? what is the effect of host population fluctuations and density dependence? etc.) As such, and to reprise our exhibition analogy, we would hope that these ideas be on permanent display for use in interdisciplinary studies.

\section{Appendices}

\section{A The Kermack-McKendrick model}

For the more mathematically interested reader, we briefly describe the Kermack-McKendrick model from 1927 in terms of the general infectivity function $\mathcal{A}$, and show how compartmental models are obtained as a special cases, where we use system (2.1) as an example.

Under the assumptions that led to system (2.1), but with transmission rate not necessarily constant, we can write

$$
\frac{d S}{d t}=-S(t) \lambda(t)
$$


where $\lambda(t)$ is the force of infection at time $t$, i.e. the fraction of the susceptible population that the infected individuals are able to meet and infect per unit of time. To express $\lambda(t)$ in familiar terms, observe that the infective individuals that have disease-age $\tau$ at time $t$, are expected to contribute infectivity $\mathcal{A}(\tau)$ towards susceptibles. How many infected individuals are there who have disease-age $\tau$ at time $t$ ? These are of course precisely those individuals that became infected themselves at time $t-\tau$. We then have to integrate over all disease-ages and hence,

$$
\lambda(t)=-\frac{1}{N} \int_{0}^{\infty} \mathcal{A}(\tau) \frac{d S}{d t}(t-\tau) d \tau .
$$

Note that $\lambda(t)$ is positive because $d S / d t$ is negative.

As an example, we derive $\mathcal{A}(\tau)$ for the compartmental disease progression as described in Section 2.1. Assume that the time periods spent in each consecutive class are exponentially distributed with some appropriate rate constant. $\mathcal{A}(\tau)$ is in this case the product of the probability of (still) being in the infective class $I$ at time $\tau$ after infection and the transmission rate at that time. Suppose we ignore the latency period and let $\gamma$ be, as before, the probability per unit of time for an infective individual to be removed. Then $e^{-\gamma \tau}$ is the probability of remaining infective after being infected for a time $\tau$, and if transmission occurs at a constant rate $\beta$ in state $I$, then $\mathcal{A}(\tau)=\beta e^{-\gamma \tau}$.

If we add a latency class $E$ with average duration $1 / \sigma$ (exponentially distributed) then similar reasoning as above leads to

$$
\mathcal{A}(\tau)=\frac{\beta \sigma}{\gamma-\sigma}\left(e^{-\sigma \tau}-e^{-\gamma \tau}\right) .
$$

As in the case without a latency period, $\int_{0}^{\infty} \mathcal{A}(\tau) d \tau=\beta / \gamma$, independent of $\sigma$. It is easy to check that with an infinitely short latency period (i.e. $\sigma \rightarrow \infty)$ we 'rediscover' $\mathcal{A}(\tau)=\beta e^{-\gamma \tau}$.

The example illustrates that we can either imagine a discrete disease-state variable with a stochastic jump process (where the holding times in the various compartments do not necessarily have to be exponentially distributed as in our examples above) to describe the progression of the disease, or a continuous disease-state variable $\tau$ with steady progression and an infectivity function $\mathcal{A}$; see Figure 11. The two representations in Figure 11 are equivalent when (A.3) holds. The $\tau$-representation is suited for a more general class of models since $\mathcal{A}$ can, in principle, be any nonnegative function.

In the special case $\mathcal{A}(\tau)=\beta e^{-\gamma \tau}$ we obtain system (2.1) from (A.2-3) by noting that the number of infective individuals at time $t$ is obtained by integrating over all recruitment into the infective class before time $t$ while taking into account the probability of being removed before $t$ is reached. 

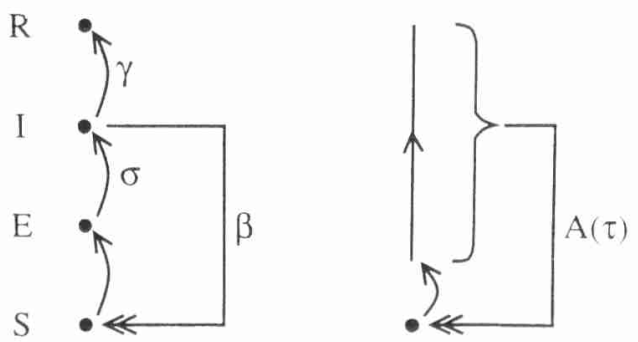

Figure 11. Metaphorical comparison of the discrete disease-state model and the continuous disease-state model.

Mathematically this means that

$$
I(t)=-\int_{-\infty}^{t} e^{-\gamma(t-\tau)} \frac{d S}{d t}(\tau) d \tau=-\int_{0}^{\infty} e^{-\gamma \tau} \frac{d S}{d t}(t-\tau) d \tau .
$$

If we substitute $\mathcal{A}(\tau)=\beta e^{-\gamma \tau}$ into $(\mathrm{A} .2)$ and use $(\mathrm{A} .4)$ then $(\mathrm{A} .1)$ is the same as the first equation in system (2.1). We obtain the differential equation for the infectives in (2.1) by differentiating (A.4) with respect to $t$.

Kermack and McKiendrick showed that the basic reproduction ratio in the general case is given by

$$
R_{0}=\int_{0}^{\infty} \mathcal{A}(\tau) d \tau
$$

For the special cases treated in this example, we rediscover $R_{0}=\beta / \gamma$. Note that $R_{0}$ is the same in this case with and without a latency class, because we neglect death in this simple model (the infected individual cannot die before reaching the infective class) and the average duration of the infective period is not affected by the latency class. See subsection 3.5 for an example where it does affect $R_{0}$.

\section{B $\quad R_{0}$ for heterogeneous models}

In the homogeneous model (2.1), $R_{0}=\beta / \gamma$. The corresponding heterogeneous model with two types of individuals is given by

$$
\begin{aligned}
\frac{d S_{i}}{d t} & =-\frac{S_{i}}{N_{i}} \sum_{j=1}^{2} \beta_{i j} I_{j} . \\
\frac{d I_{i}}{d t} & =\frac{S_{i}}{N_{i}} \sum_{j=1}^{2} \beta_{i j} I_{j}-\gamma_{i} I_{i},
\end{aligned}
$$

$i \in\{1,2\}, N_{i}=S_{i}+I_{i}$. Let us interpret, for example, $i=1$ as female individuals and $i=2$ as males. In the same heuristic way as for (2.1), we could then for the heterogeneous system (B.1) calculate a quantity $m_{11}$ say, which gives the expected number of new male cases that are caused by one 
male infected individual in a population consisting only of male susceptibles. In the same way one could calculate quantities $m_{12}, m_{21}$ and $m_{22}$ with obvious interpretations. So, instead of the single number we had before, we now have four different numbers. The basic reproduction ratio for the heterogeneous population will be some average of these four $m_{i j}$ 's, the question we want to answer is: how should we take this average in order to arrive at a quantity which possesses the threshold property that an epidemic can develop if it is bigger than one, whereas no epidemic will develop if it is less than one?

By just applying the biological interpretation of the $m_{i j}$ 's we can obtain expressions for them from the model equations. We arrange the four expressions into a matrix

$$
M:=\left(\begin{array}{ll}
m_{11} & m_{12} \\
m_{21} & m_{22}
\end{array}\right)=\left(\begin{array}{ll}
\beta_{11} / \gamma_{1} & \beta_{12} / \gamma_{2} \\
\beta_{21} / \gamma_{1} & \beta_{22} / \gamma_{2}
\end{array}\right) .
$$

If we now regard generations of infected individuals (i.e. the initially infected animals are the first generation, all animals that become infected through contacts with members of the first generation are collectively called the second generation, etc.), then the matrix $M$ relates the number of infected male and female animals in the present generation, represented by a vector $X=$ $\left(x_{1}, x_{2}\right)^{T}$, to the respective numbers in the next generation given by the vector $M X$. One can easily see that this is a good interpretation of the matrix $M$ because, for example, the first component of $M X$ is given by $x_{1} \beta_{11} / \gamma_{1}+$ $x_{2} \beta_{12} / \gamma_{2}$, which is precisely the total number of new male cases that we expect will become infected by the combined 'efforts' of $x_{1}$ infected males and $x_{2}$ infected females. It is clear that the matrix $M$ (called the nextgeneration matrix) contains information that can answer our question. Note that, because we are dealing with biological quantities, all entries of the nextgeneration matrix will be non-negative. $M$ therefore is a positive matrix.

Let $\omega>\mu$, with $\omega>0$, be the two eigenvalues of the positive matrix $M$ with eigenvectors $W$ and $U($ so, $M W=\omega W$, and $M U=\mu U)$, then we can write any vector $X=\left(x_{1}, x_{2}\right)^{T}$ as a linear combination of $W$ and $U$, $X=a_{1} W+a_{2} U$, where the $a_{i}$ depend on $X$. If we apply the matrix $M$ to both sides we obtain

$$
\begin{aligned}
M X & =M\left(a_{1} W+a_{2} U\right)=a_{1} M W+a_{2} M U \\
& =a_{1} \omega W+a_{2} \mu U
\end{aligned}
$$

and applying $M n$ times leads to

$$
\begin{aligned}
M^{n} X & =a_{1} \omega^{n} W+a_{2} \mu^{n} U \\
& =\omega^{n}\left(a_{1} W+a_{2}\left(\frac{\mu}{\omega}\right)^{n} U\right) .
\end{aligned}
$$

From this last expression we see that the behaviour of the infected population of males and females will, after many generations, be determined by the dominant eigenvalue $\omega$ of $M$ and its eigenvector. Indeed, for $n \rightarrow \infty,(\mu / \omega)^{n} \rightarrow 0$ 
and

$$
M^{n} \mathrm{X} \approx a_{1} \omega^{n_{1}} W .
$$

So, if $\omega>1$, the consecutive generations will increase in size, whereas for $\omega<1$ the generation sizes will approach zero. After many generations, each generation will be $v$ bigger than the previous one, and the distribution of infected individuals over males and females will become fixed and is given by W.

These properties of $\omega$ motivate the following definition of the basic reproduction ratio $R_{0}$ in a heterogeneous population

$$
R_{0}=\omega=\text { the dominant eigenvalue of the matrix } M \text {. }
$$

Thus, $R_{01}$ is defined on a generation basis. One can show that $R_{0}>1$ indeed leads to an increase in the infected population in real time and that $R_{0}<1$ leads to a decrease in real time.

For the example given in Section 5 (system (5.1)) we find that the nextgeneration matrix $M$ has elements $m_{i j}=\beta_{i j} /\left(\rho_{j}+\alpha_{j}\right)$ (expected number of new $i$-cases caused per unit of time by $j$-infective, multiplied by average (duration of infectious period) and under the special parameter assumptions listed in Section 5, we find

$$
M=\left(\begin{array}{cc}
0 & \beta_{12} / \alpha_{2} \\
\beta_{21} / \rho_{1} & 0
\end{array}\right)
$$

and the dominant eigenvalue can easily be calculated from the characteristic equation $\operatorname{det}(M-\lambda I d)=0$, where $I d$ is the identity-matrix. We find

$$
h_{0}=\sqrt{\frac{\beta_{21}}{\alpha_{2}} \frac{\beta_{12}}{\rho_{1}}} .
$$

The above ideas can be found in Hethcote and Yorke (1984). If one recognises $n$ (sub)groups of animals, then $M$ will become an $n \times n$-matrix with the element $m_{2 j}$ being the expected number of new cases among members of group $i$ that are caused by infected members of group $j . R_{0}$ is again the dominant eigenvalue of $M$ (Hethcote and Yorke 1984). In general, if there are $n$ types of individuals, calculating $R_{0}$ would mean first calculating the matrix $M$, and then numerically determining the dominant eigenvalue. In De Jong et al. (1994) an explicit algorithm is given to calculate the matrix $M$ for multigroup models from basic model ingredients and assumptions.

In one special case it is easy to calculate $R_{0}$, in fact even an explicit formula can be given. The assumption that makes this possible is that every element $m_{i j}$ of $M$ can be written as

$$
m_{i j}=f_{i} g_{j}
$$


which means that contributions to $m_{i j}$ by the susceptible individual (type i) and that of the infective individual (type $j$ ) are separated. This is called separable mixing (the well-known proportionate mixing is a special case of this when $g_{j}=c f_{j}$, where $c$ is a constant). The biological interpretation of separable mixing is that the types of individuals that can be infected by a given infective animal are independent of that animal's own type. Under that assumption

$$
R_{0}=\sum_{i=1}^{n} m_{i 2}
$$

being the sum of the elements on the diagonal of $M$. In the case of model (B.1), this leads to

$$
R_{0}=\frac{\beta_{11}}{\gamma_{1}}+\frac{\beta_{22}}{\gamma_{2}}
$$

The mathematics of defining and calculating $R_{0}$ in populations with any given heterogeneity structure can be found in Diekmann et al. (1990) and Heesterbeek (1992).

\section{Acknowledgements}

We thank the Isaac Newton Institute for the opportunity to work there for 6 months and for the organisational inspiration to put us in the same office. We would like to thank Andy Dobson, Gary Smith, Herb Hethcote, Mart De Jong, Frank Ball and Bryan Grenfell for stimulating discussions. JAPH was financially supported by the Netherlands Organisation for Scientific Research NWO, the Royal Society and SERC Grant GR G59981; MGR received financial assistence from a Trimble Agricultural Research Fellowship and MSD-AgVet Ltd.. and an extended period of leave from the New Zealand Pastoral Agricultural Research Institute Ltd.

\section{References}

Anderson, R.M. (1982a) 'Fox rabies'. In Anderson (1982b).

Anderson, R.M. (ed.) (1982b) The Population Dynamics of Infectious Diseases: Theory and Applications, Chapman and Hall, London.

Anderson, R.M., Jackson, H.C., May, R.M. and Smith, A.D.M. (1981) 'Population dynamics of fox rabies in Europe', Nature 289, 765-770.

Anderson, R.M., and May, R.M. (1979) 'Population biology of infectious diseases: Part I', Nature 280, 361-367.

Anderson, R.M., and May, R.M. (1991) Infectious Diseases of Humans, Dynamics and Control, Oxford University Press, Oxford. 
Anderson, R.M. and Trewhella, W. (1985) 'Population dynamics of the badger (Meles meles) and the epidemiology of bovine tuberculosis (Mycobacterium bovis)', Phil. Trans. R. Soc. B 310, 327-381.

Bacon, P.J. (ed.) (1985) Population Dynamics of Rabies in Wildlife, Academic Press, London.

Bailey, N.J.T. (1975) The Mathematical Theory of Infectious Diseases and Its Application, Griffin, London.

Baltensweiler, W. (1968) 'The cyclic population dynamics of the grey larch tortrix, Zeiraphera griseana (Hubner) (= Semasia diniana (Guenee))', Symp. $R$. Entomol. Soc., Lond. 4, 88-97.

Bartlett, M.S. (1960) Stochastic Population Models in Ecology and Eprdemiology, Methuen, London.

Becker, N. (1989) Analysis of Infectious Disease Data, Chapman and Hall, London.

Bolker, B.M. and Grenfell, B.T. (1993) 'Chaos and biological complexity in measles dynamics', Proc. Roy. Soc. London B 251, 75-81.

Bowers, R.G., Begon, M. and Hodgkinson, D.E. (1993): Host-pathogen population cycles in forest insects? Lessons from simple models reconsidered. Oikos 67 , $529-538$.

Busenberg, S.N and Cooke, K. (1993) Vertically Transmitted Diseases, Models and Dymamics, Jecture Notes in Biomathematics 23, Springer-Verlag, Berlin.

Busenberg, S.N. and van den Driessche, P. (1990) 'Analysis of a disease transmission model in a population with varying size', J. Math. Biol. 28, 257-270.

Coyne, M. et al. (1989) 'Mathematical models for the population biology of raccoon rabies in the mid-atlantic states', Am. J. Vet. Res. 50, 2148-2154.

Cubb, S.L. (1986) 'Avian pox in cage and aviary birds'. In Zoo and Wild Animal Medicine, second edition, M.E. Fowler (ed.), W.B. Saunders Co., Philadelphia, 214-219.

De Jong, M.C.M., Diekmann, O. and Heesterbeek, J.A.P. (1994) 'The computation of $R_{0}$ for discrete-time epidemic models with dynamic heterogeneity', Math. Biosci. 119, 97-114.

De Jong, M.C.M., Diekmann, O. and Heesterbeek, J.A.P. (1995) 'How does infection-transmission depend on population size?'. In Epidemic Models: their Structure and Relation to Data, Denis Mollison (ed.), Cambridge University Press, Cambridge, to appear.

Diekmann, O. (1991) 'Modelling infectious diseases in structured populations'. In Ordinary and Partial Differential Equations, vol. III, B.D. Sleeman and R.J. Jarvis (eds.), Pitman RNiMS 254, Longman, Harlow, 67-79.

Diekmann, O., de Jong, M.C.M., de Koeijer, A.A. and Reijnders, P. (1994) 'The force of infection in growing populations: a modelling problem'. Submitted to Proc. 2nd European Conf. on Math. Applied to Biol. and Med., Lyon (1993). 
Diekmamn, O., Heesterbeek, J.A.P. and Metz, J.A.J. (1990) 'On the definition and the computation of the basic reproduction ratio $R_{0}$ in models for infectious diseases in heterogeneous populations', J. Math. Biol. 28, 365-382.

Diekmann, O., Heesterbeek, J.A.P. and Metz, J.A.J. (1995) 'The legacy of Kermack and McKendrick'. In Epidemic Models: their Structure and Relation to Data, Denis Mollison, (ed.), Cambridge University Press, Cambridge, to appear.

Diekmann, O. and Kretzschmar, M. (1991) 'Patterns in the effects of infectious diseases on population growth', J. Math. Biol. 29, 539-570.

Dietz, K. (1982) 'Overall population patterns in the transmission cycle of infectious disease agents'. In Population Biology of Infectious Diseases, R.M. Anderson, R.M. May (eds.), Springer-Verlag, Berlin, 87-102.

Dietz, K. (1992) 'The estimation of the basic reproduction number for infectious diseases', Stat. Meth. Med. Rescarch 2, 23-41.

Dwyer, G. and Elkington, J.S. (1993) 'Using simple models to predict virus epizootics in gypsy moth populations', J. Anim. Ecol. 62, 1-11.

Gabriel, J.-P., Lefèvre, C. and Picard, P. (eds.) (1990) Stochastic Processes in Epidemic Theory, Lecture Notes in Biomathematics, 86, Springer, Berlin.

Gabriel, J.-P., Lefèvre, C., Picard, P. and Jacquez, J.A. (eds.) (1993) Stochastic Modclling for Infectrous Diseases, Special issue of Math. Biosci. 117.

Graham, D.L. and Halliwell, W.H. (1986) 'Viral diseases of birds of prey'. In Zoo and Wild Animal Medicine, second edition, M.E. Fowler (ed.), W.B. Saunders Co., Philadelphia, 214-219

Greenhalgh, D. and Das, R. (1993) 'An SIR epidemic model with a contact rate depending on population density'. Preprint.

Grenfell, B.T. (1992) Chance and chaos in measles dynamics', J. Roy. Stat. Soc. B 54, 383-398.

Grenfell, B.T., Longergan, M.E. and Harwood, J. (1992) 'Quantitative investigations of the epidemiology of phocine distemper virus (PDV) in European common seal populations', Sci. Tot. Environ. 115, 15-28.

Heesterbeek, J.A.P. (1992) $R_{0}$, PhD Thesis, University of Leiden.

Heesterbeek, J.A.P. and Metz, J.A.J. (1993) 'The saturating contact rate in marriage and epidemic models', J. Math. Biol. 31, 529-539.

Hethcote, H.W. (1976) 'Qualitative analysis for communicable disease models', Math. Biosci. 28, 335-356.

Hethcote, H.W. (1994) 'A thousand and one epidemic models'. In Frontiers in Theoretical Biology, S.A. Levin (ed.), Lecture Notes in Biomathematics, 100, Springer, Berlin.

Hethcote, H.W. and Levin, S.A. (1989) 'Periodicity in epidemic models'. In Applied Mathematical Ecology, S.A. Levin, T.G. Hallam, L.J. Gross (eds.), SpringerVerlag, Berlin, 193-211. 
Hethcote, H.W and Yorke, J.A. (1984) Gonorhea: Transmission Dynamics and Control. Lecture Notes in Biomathematics 56, Springer-Verlag, Berlin.

Hethcote, II W and Van Ark, J.W (1992) Modellang HIV Transmission and AIDS in the Unted States, Lecture Notes in Biomathematics 95, Springer-Verlag, Berlin.

Kermack. W.O. and Mckendrick, A.G. (1927) 'Contributions to the mathematical theory of epidemics, part I', Proc. Roy. Soe. Edin. A 115, 700721.

Linhart S.B. of al. (1991) Formulation and evaluation of baits for oral rabies vaccination of raccoons (Procyon lotor)', J. Wild. Dis. 27, 21-33.

Liu, W.M. (1992) Models of recurrent outbreaks of infectious diseases:. In Procredings 3rd International Conference on Mathematical Population Dynames, Pau, 1992, to appear.

Metz, J.A.J. and van den Bosch, F. (1995) 'Velocities of epidemic spread'. In Emdemic Models: their Structure and Relation to Data, Denis Mollison (ed.), Cambridge University Press, Cambridge, to appear.

Mollison, D., Scalia-Tomba, G. and Jacquez, J.A. (eds.) (1991) Spread of Epidemics: Stochastic Modelling and Data Analysis, special issue of Math. Biosci. 107.

Onstad, D.W and Maddox, J.V. (1990) Simulation model of Trhohum confusum and its pathogen Nosema whiter', Ecol. Modell. 51, 143-160.

Peterson, M.J.. Grant, W.E. and Davis, D.S. (1991) 'Simulation of host parasite interactions within a resource management framework: impact of brucellosis on bison population dynamics. Ecol. Modell. 54, 299-320.

Roberts, M.C. (1992) The dynamics and control of bovine tuberculosis in possums, IMA J. Wath. Appl. Med. Brol. 9, 19-28.

Roherts, M.G. and Heesterbeek, J.A.P. (1993) 'Bluff your way in epidemic models', Trends in Microbiol. 1, 343-348.

Ross, R. and Hudson, H.P. (1917) An application of the theory of probabilities to the study of a priori pathometry. part III', Proc Roy. Soc Lond. A 43 , $225-240$.

Schenzle, D. and Dietz, K. (1987) 'Critical population sizes for endemic virus transmission:. In Räumliche Persistenz und Diffusion von Krankheaten, W. Fricke and E. Hinz (eds.), Heidelberger Geographische Arbeiten, 83, Heidelberg, 3142.

Snith, G. and Dobson, A.P. (1992) 'Sexually transmitted diseases of animals', Parasit. Today 8, 159-166.

Swart, J.H. (1989) 'Hopf bifurcation and stable limit cycle behaviour in the spread of infections diseases, with special application to fox rabies', Math. Biosci. 95 , 199-207.

Thieme, H.R. (1992) 'Epidemic and demographic interaction in the spread of potentially fatal diseases in growing populations', Math. Broscr. 111, 99-130.

Zhou, J. and Hethcote, H.W. (1994) 'Population size dependent incidence in models for diseases without immunity', J. Math. Biol, 32, 809-834. 